\title{
Vitamin B12 and Folic Acid Levels in Known Coronary Artery Disease Patients
}

\section{Ukendiran.D ${ }^{1}$, Sri Ram.V ${ }^{2}$, Renuka.P ${ }^{3}$, V.E. Dhandapani ${ }^{4}$, S. Aishwarya ${ }^{5}$, V.M. Vinodhini*6}

${ }^{1}$ Senior laboratory technologist, Department of Biochemistry, Panimalar Medical College Hospital and Research Center, Tiruvallur-600123, Tamilnadu, India.

${ }^{2}$ Associate professor, Department of Cardiology, SRM Medical College Hospital and Research Center, SRM Institute of Science and Technology, Kattankulathur, Kanchipuram, Chennai, Tamilnadu, India - 603203.

${ }^{3}$ Professor, Department of Biochemistry, SRM Medical College Hospital and Research Center, SRM Institute of Science and Technology, Kattankulathur, Kanchipuram, Chennai, Tamilnadu, India - 603203.

${ }^{4}$ HOD and Professor, Department of Cardiology, SRM Medical College Hospital and Research Center, SRM Institute of Science and Technology, Kattankulathur, Kanchipuram, Chennai, Tamilnadu, India - 603203.

${ }^{5}$ Research scholar, Department of Biochemistry, SRM Medical College Hospital and Research Center, SRM Institute of Science and Technology, Kattankulathur, Kanchipuram, Chennai, Tamilnadu, India - 603203.

${ }^{* 6}$ HOD and Professor, SRM Medical College Hospital and Research Center, SRM Institute of Science and Technology, Kattankulathur, Kanchipuram, Chennai, Tamilnadu, India 603203.

${ }^{1}$ Email: yugendiran97@gmail.com, ${ }^{2}$ Email: sriramv@srmist.edu.in, ${ }^{3}$ Email: renukap@srmist.edu.in, ${ }^{4}$ Email:dhandape@srmist.edu.in, ${ }^{5}$ Email: as4826@srmist.edu.in,

${ }^{* 6}$ Email: vinodhiv1@srmist.edu.in.

\footnotetext{
*V.M Vinodhini, Professor and Head, Department of Biochemistry, SRM Medical College Hospital and Research Center, SRM Institute of Science and Technology, Kattankulathur, Kanchipuram, Chennai, Tamilnadu, India - 603203. Email id: vinodhiv1@ srmist.edu.in.
} 


\begin{abstract}
Vitamin $B 12$ and folic acid are water soluble vitamins playing a vital role in various biological processes, where their deficiency leading to hyperhomocysteinemia are closely related to the development of cardio vascular risk. This case-control study included a total of 60 participants; 30 with confirmed coronary artery disease (CAD) and 30 were age and sex matched healthy controls. Fasting blood samples were collected and analyzed for biochemical parameters. Statistical analysis was done using SPSS software. A p value <0.05 was considered statistically significant. Vitamin B12 levels were found to be significantly lower in $C A D$ patients than the controls. Based on the results, $50 \%$ of CAD patients had vitamin B12 deficiency. Vitamin B12 was positively associated with HDL-c and negatively with TC, TGL, $L D L-c, V L D L-c, R L P-c$, Non-HDL-c, TG/HDL-c. The study concludes that low levels of vitamin $B 12$ and folic acid are related with risk factors of coronary artery disease.
\end{abstract}

Key words: Coronary artery disease, Folic acid, Risk factors, Vitamin B12, Hyperhomocysteinemia.

\title{
Introduction
}

Coronary artery disease (CAD) is a major public health problem and a leading cause of morbidity and mortality around the world. Dysfunctional endothelium and impaired endothelium dependent relaxation of blood vessels appears to be the initiating factors in the development of atherosclerosis [1].

Folic acid and vitamin B12 are B-group vitamins that play an essential role in many biological processes. These water-soluble vitamins, are usually not retained by the body with the exception of vitamin B12 which is stored in the liver. As these vitamins are not synthesized by the human body in sufficient quantity, they have to be derived from the diet [2].

In recent times there has been a lot of interest regarding the role of folic acid supplementation in the prevention of several disorders [3]. Folic acid was found to have a favorable effect in the improvement of endothelial dysfunction [4]. The predominant form of folic acid in circulation is 5- MTHF (5- methyl tetra hydro folate). 5-MTHF exhibit anti atherogenic effect by the following mechanisms which includes (i) decrease in homocysteine level (ii) antioxidant effect (iii) interaction with endothelial nitric oxide synthase (eNOS) and (iv) availability of nitric oxide [3]. It has been found that folic acid has the ability to revert dysfunction of eNOS [5].

Vitamin B12 is found in nature as cobalamins. It is composed of a corrin ring namely cobalt,5,6 dimethyl benzimidazole, ribose and an aminopropanol group. Cyanocobalamin is the most common available drug form of vitaminB12, characterized by having a cyanide group in one of its axial positions [6]. Vitamin B12 acts as a co-factor for the enzyme methionine synthetase in the reaction of methylation of homocysteine. Therefore, folic acid and vitamin B12 deficiency can cause reduction in methylene tetrahydro-folic acid reductase (MTHFR) activity, leading to decrease in methionine synthesis and homocysteine accumulation [7]. Deficiency of vitamin B12 and folic acid leading onto hyperhomocysteinemia are closely related to the development of cardio vascular risk factor [1].

The prevalence of folate and vitamin B12 deficiency among CAD patients is found to be widely variable across different population groups. Therefore, this study was planned to 
investigate the levels of serum vitamin B12 and folic acid in patients suffering from coronary artery disease (CAD) compared to healthy subjects and to correlate their levels with the traditional cardiac risk factors.

\section{Materials and Methods}

This case control study was conducted in department of Cardiology, SRM Medical College Hospital and Research Center, Kattankulathur, Kanchipuram, Chennai, Tamilnadu, India. The study consisted of 60 participants out of which 30 were patients with angiographically proven CAD in the age group of 30-70 years of both genders. CAD was defined as angiographically proven coronary disease with $>50 \%$ stenosis of at least 1 major epicardial coronary artery.

Age and sex matched 30 apparently healthy volunteers attending the master health check-up programme of SRM Medical College Hospital and Research Center, Kattankulathur, Kanchipuram, Chennai, Tamilnadu, India formed the control group. Presence of renal, hepatic disease, endocrine disorders, malabsorption, pregnancy, treatment with antifolate agents such as methotrexate and also folic acid and vitamin B12 supplementation were considered as exclusion factors.

A fasting venous blood sample was drawn after 12 hours of overnight fasting. Plasma glucose was measured by hexokinase method, serum triglycerides (TGL) by glycerol peroxidase, total cholesterol (TC) by cholesterol oxidase, low-density lipoprotein (LDL-c) and high-density lipoprotein (HDL-c) by direct method in Beckman coulter auto analyzer. VLDL-c by calculation, RLP-c was calculated using the formula TC- (HDL-c + LDL-c). Serum vitamin B12 and Folic acid were measured by competitive binding assay in VITROS ECIQ immuno analyzer by Ortho Clinical Diagnostics.

The study protocol was approved by the Institutional Ethics Committee (ECN: 1867(A)IEC/2019) and informed written consent was taken from all the participants.

\section{Statistical analysis}

Data were analyzed using statistical package for the social science (SPSS) version 25. The continuous variables are represented as mean \pm standard deviation (SD). Categorical variables are expressed as frequencies and percentages. Independent student's t-test was used to compare the mean levels of age, BMI and analyzed biochemical parameters between CHD patients and healthy participants. One way ANOVA was used for comparison of analyzed biochemical parameters between vitamin B12 deficient CAD, vitamin B12 Non-deficient CAD and control groups. The correlation coefficient of CAD patients with each of the variables was assessed using Pearson's correlation. For all statistical tests, a $\mathrm{p}$ value $<0.05$ was taken to indicate a significant difference.

\section{Results}

Among the 30 patients with CAD, $25(83 \%)$ were males and $5(17 \%)$ were females. The mean age of the CAD patients was found to be $52 \pm 6.6$ years (range $30-70$ years). The mean BMI 
was found to be $25.5 \pm 2.86 \mathrm{~kg} / \mathrm{m}^{2}$. Among the CAD patients $10 \%$ were obese, $60 \%$ were diabetic, $30 \%$ were smokers, $11 \%$ were hypertensive, $3 \%$ were on a vegetarian diet and $36 \%$ had hyper-cholesterolemia. Considering the results of coronary angiogram of CAD patients, $15(50 \%)$ had single vessel disease (SVD), 12(40\%) had double vessel disease (DVD) and 3 $(10 \%)$ had triple vessel disease (TVD). Among the control group, 25 (83\%) were males and $5(17 \%)$ were females with a mean age of $48 \pm 14$. 2 years. The BMI of this control group was $24 \pm 2.95$. All of the control participants were non-vegetarians.

To study the effect of age on CAD we divided the case and control subjects into four groups from (30-40), (41-50), (51-60), (61-70) years. We found that a greater number of CAD patients are in the age group (50-60 years) (Table 1$)$.

Table 1. Age distribution of CAD patients and controls

\begin{tabular}{|l|l|l|l|l|l|}
\hline \multicolumn{2}{|c|}{} & \multicolumn{4}{|l|}{ Groups } \\
\cline { 3 - 6 } \multicolumn{2}{|l|}{ CAD } & Control \\
\cline { 3 - 6 } \multicolumn{2}{|l}{} & Numbers & $\%$ & Numbers & $\%$ \\
\hline \multirow{2}{*}{$\begin{array}{l}\text { Age } \\
\text { groups } \\
\text { years }\end{array}$} & $\mathbf{3 0 - 4 0}$ & 2 & 7 & 9 & 30 \\
\cline { 2 - 6 } & $\mathbf{4 1 - 5 0}$ & 10 & 33 & 4 & 13 \\
\cline { 2 - 6 } & $\mathbf{5 1 - 6 0}$ & 16 & 53 & 8 & 27 \\
\cline { 2 - 6 } & $\mathbf{6 1 - 7 0}$ & 2 & 7 & 9 & 30 \\
\hline
\end{tabular}

The mean plasma glucose and serum levels of total cholesterol, triglycerides, HDL-c, LDL-c, VLDL, Non-HDL-c, TC/HDL ratio, TG/HDL-c ratio, vitamin B12 and folic acid of both case and control groups are listed in (Table 2). There was a significant difference found in FPG, TG, HDL-c, LDL-c, VLDL-c, Non-HDL-c, TC/HDL-c, TG/HDL-c, LDL/HDL-c and vitamin B12 between $\mathrm{CAD}$ patients and controls.

Table 2. Baseline characteristics of control and CAD group

\begin{tabular}{|l|l|l|l|}
\hline Variables & CAD group, N=30 & $\begin{array}{l}\text { Control group, } \\
\text { N=30 }\end{array}$ & p value \\
\hline Clinical factors & \multicolumn{3}{l|}{} \\
\hline Age $($ years $)$ & $52 \pm 6.6$ & $48 \pm 14.2$ & 0.1671 \\
\hline BMI $\left(\mathrm{kg} / \mathrm{m}^{2}\right)$ & $25.5 \pm 2.86$ & $24.8 \pm 2.9$ & 0.3455 \\
\hline FBG $(\mathrm{mg} / \mathrm{dL})$ & $174 \pm 62.2$ & $106 \pm 9.0$ & $0.0001^{*}$ \\
\hline TC $(\mathrm{mg} / \mathrm{dL})$ & $181 \pm 48.6$ & $160 \pm 24.93$ & 0.0396 \\
\hline TG $(\mathrm{mg} / \mathrm{dL})$ & $183 \pm 87.4$ & $86.5 \pm 29.13$ & $0.0001^{*}$ \\
\hline HDL-C $(\mathrm{mg} / \mathrm{dL})$ & $39 \pm 9.6$ & $46 \pm 10.41$ & $0.0089^{*}$ \\
\hline LDL-C (mg/dL) & $128 \pm 35.8$ & $106 \pm 24.38$ & $0.0073^{*}$ \\
\hline VLDL-C (mg/dL) & $36.5 \pm 17.5$ & $17.3 \pm 5.82$ & $0.0001^{*}$ \\
\hline $\begin{array}{l}\text { Non-HDL-C } \\
(\mathrm{mg} / \mathrm{dL})\end{array}$ & $142 \pm 42.6$ & $114 \pm 27.4$ & $0.0037^{*}$ \\
\hline RLP-C (mg/dL) & $14 \pm 18$ & $8.1 \pm 8$ & 0.1063 \\
\hline
\end{tabular}




\begin{tabular}{|c|c|c|c|}
\hline TC/HDL-c & $4.7 \pm 1$ & $3.64 \pm 0.98$ & $0.0001 *$ \\
\hline TG/HDL-c & $4.77 \pm 2.27$ & $2 \pm 0.87$ & $0.0001 *$ \\
\hline LDL/HDL-c & $3.35 \pm 0.9$ & $2.43 \pm 0.78$ & $0.0001 *$ \\
\hline $\begin{array}{ll}\text { Vitamin } & \text { B12 } \\
(\mathrm{pg} / \mathrm{ml}) & \end{array}$ & $242 \pm 98$ & $333.7 \pm 197.1$ & $0.0261^{*}$ \\
\hline Folic acid (ng/ml) & $4.68 \pm 3.6$ & $5.03 \pm 3.37$ & 0.6989 \\
\hline
\end{tabular}

In both the CAD as well as the control groups, the level of vitamin B12 was lesser in males than females but this difference was not statistically significant. In both the CAD as well as the control groups, folic acid levels in males was lesser than females but in control group it was found to be significant. (Table.3)

TABLE 3. Concentration of serum vitamin B12 and Folic acid levels in study population (mean $\pm \mathrm{SD}$ ) based on the gender.

\begin{tabular}{|l|l|l|l|l|l|l|}
\hline \multicolumn{2}{|l|}{ Study Population } & $\begin{array}{l}\text { Number } \\
(\%)\end{array}$ & $\begin{array}{l}\text { Vitamin B12 } \\
\text { pg/ml }\end{array}$ & P value & $\begin{array}{l}\text { Folic acid } \\
\text { ng/ml }\end{array}$ & $\begin{array}{l}\text { P } \\
\text { value }\end{array}$ \\
\hline \multirow{2}{*}{ Control } & male & $25(83)$ & $299.2 \pm 103.4$ & 0.05 & $5.1 \pm 3.4$ & $0.04^{*}$ \\
\cline { 2 - 4 } & female & $5(17)$ & $506 \pm 415$ & & $5.67 \pm 2.6$ & \\
\hline \multirow{2}{*}{ Case } & male & $25(83)$ & $241.12 \pm 104$ & 0.05 & $3.6 \pm 1.3$ & 0.24 \\
\cline { 2 - 4 } & female & $5(17)$ & $248.2 \pm 61.7$ & & $4.0 \pm 1.5$ & \\
\hline
\end{tabular}

The Pearson's correlation coefficient of vitamin B12 of CAD categories with each of the laboratory parameters were analyzed. Vitamin B12 was found to be positively correlated with fasting plasma glucose, HDL-c, TC/HDL-c and LDL/HDL-c and negatively correlated with TC, RLP-c, TGL, LDL-c, VLDL-c, Non-HDL-c, TG/HDL-c. (Table 4)

Table 4. Pearson's correlation coefficient between vitamin $B 12$ and other laboratory parameters in CAD patients.

\begin{tabular}{|l|l|l|}
\hline $\begin{array}{l}\text { Correlation of vitamin } \\
\text { B12 } \text { with }\end{array}$ & R VALUE & P VALUE \\
\hline FPG $(\mathrm{mg} / \mathrm{dl})$ & 0.3618 & $0.0494^{*}$ \\
\hline TC $(\mathrm{mg} / \mathrm{dl})$ & -0.0018 & 0.9958 \\
\hline TGL $(\mathrm{mg} / \mathrm{dl})$ & -0.1234 & 0.517 \\
\hline HDL $-\mathrm{c}(\mathrm{mg} / \mathrm{dl})$ & 0.021 & 0.9122 \\
\hline LDL-c $(\mathrm{mg} / \mathrm{dl})$ & -0.0225 & 0.9081 \\
\hline VLDL-c $(\mathrm{mg} / \mathrm{dl})$ & -0.1234 & 0.1234 \\
\hline RLP-c $(\mathrm{mg} / \mathrm{dl})$ & -0.0415 & 0.827 \\
\hline Non-HDL $(\mathrm{mg} / \mathrm{dl})$ & -0.0068 & 0.974 \\
\hline TC/HDL & 0.0871 & 0.6471 \\
\hline
\end{tabular}




\begin{tabular}{|c|c|c|}
\hline LDL/HDL & 0.0518 & 0.785 \\
\hline TG/HDL & -0.0693 & 0.7171 \\
\hline Folic acid ng/ml & 0.594 & $0.0005 *$ \\
\hline
\end{tabular}

The CAD patients were stratified into two groups on their vitamin B12 status. Among them, $15(50 \%)$ were vitamin B12 deficient ( $<200 \mathrm{pg} / \mathrm{ml})$. One way ANOVA comparison showed significant relation with control group. (Table 5)

Table 5. Comparison of laboratory parameters between the vitamin B12 deficient CAD, vitamin B12 Non-deficient of CAD group and control groups

\begin{tabular}{|l|l|l|l|l|}
\hline Variable & $\begin{array}{l}\text { Case deficient } \\
\text { B12 }\end{array}$ & $\begin{array}{l}\text { Case non- } \\
\text { deficientB12 }\end{array}$ & Control & P value \\
\hline FPG mg/dl & $160.6 \pm 53.2$ & $187.6 \pm 71.2$ & $106 \pm 9$ & $0.00 *^{*}$ \\
\hline TC mg/dl & $187 \pm 53.5$ & $175.4667 \pm 46$ & $160 \pm 24$ & 0.86 \\
\hline TG mg/dl & $197 \pm 101.30$ & $168 \pm 75.3$ & $86.5 \pm 29.1$ & $0.001^{*}$ \\
\hline HDL-c mg/dl & $40.1 \pm 9.15$ & $38.3 \pm 10.66$ & $46 \pm 10$ & $0.043^{*}$ \\
\hline LDL-c mg/dl & $131 \pm 40.5$ & $125.2 \pm 32.9$ & $106 \pm 24.4$ & $0.025^{*}$ \\
\hline VLDL-c mg/dl & $39.43 \pm 20.3$ & $33.6 \pm 15$ & $17.3 \pm 5.8$ & $0.001^{*}$ \\
\hline $\begin{array}{l}\text { Non-HDL-c } \\
\text { mg/dl }\end{array}$ & $146.9 \pm 46$ & $137.1 \pm 40.9$ & $114 \pm 27.4$ & $0.012^{*}$ \\
\hline RLP-c mg/dl & $16.3 \pm 14$ & $18.6 \pm 16$ & $8.6 \pm 7.8$ & $0.025^{*}$ \\
\hline TC/HDL-c & $4.6 \pm 0.8$ & $4.7 \pm 1$ & $3.6 \pm 0.98$ & $0.001^{*}$ \\
\hline LDL-c/HDL-c & $3.2 \pm 0.83$ & $3.42 \pm 1$ & $2.4 \pm 0.7$ & $0.004^{*}$ \\
\hline TG/HDL-c & $5.0 \pm 2$ & $4.5 \pm 2.1$ & $2 \pm 0.9$ & $0.001^{*}$ \\
\hline $\begin{array}{l}\text { Vitamin B12 } \\
\text { pg/ml }\end{array}$ & $180 \pm 13$ & $304 \pm 111$ & $333 \pm 197$ & $0.008^{*}$ \\
\hline Folic acid ng/ml & $3.2 \pm 0.7$ & $4.3 \pm 1.6$ & $5.2 \pm 3.3$ & $0.04^{*}$ \\
\hline
\end{tabular}

Tc: Total cholesterol, TG: Triglyceride, LDL-c: Low density lipoprotein cholesterol, HDL-c: High density lipoprotein cholesterol, VLDL-c Very low-density lipoprotein cholesterol, RLP-c: Remnant lipoprotein cholesterol. * significant $(\mathrm{p}<0.05)$.

The CAD patients were further stratified based on the angiographic findings as $15(50 \%)$ SVD, $12(40 \%)$ DVD and $3(10 \%)$ TVD. It was observed that $60 \%$ of SVD and $50 \%$ of DVD patients were vitamin B12 deficient and none of the TVD patients were found to be vitamin B12 deficient.

In order to evaluate the risk of vitamin B12 deficiency for CAD group other than the known risk factors such as diabetes, hyperlipidemia, hypertension, smoking and family history, logistic regression test was performed. Results showed that odds of developing CAD due to vitamin B12 deficiency was 5 times more than the odds of developing CAD without vitamin B12 deficiency CAD. (OR = 5, 95\% CI: 1.52-16.56 p=0.0261). 


\section{Discussion}

Vitamin B12 and folic acid have been shown to play a significant role in lowering the risk of coronary artery disease [8]. Robinson et al identified reduced levels of folic acid and vitamin B12 in patients with CAD [9]. Vitamin B12 and folic acid are involved in regulation of homocysteine by remethylation reaction. Methyl-tetra-hydrofolic acid (MTHF), a folic acid derivative provides methyl group for remethylation of homocysteine. Vitamin B12 acts as a cofactor for methionine synthase that catalyses the reaction [10].

Several studies have indicated homocysteine as an independent risk factor for coronary artery disease. Clark et al [11] and Humphery et al [12] showed an association between hyperhomocysteinemia and increase in risk of CAD [12]. A study by Roman Pawlak, showed that each $5-\mu \mathrm{mol} / \mathrm{L}$ increase above $10 \mu \mathrm{mol} / \mathrm{L}$ of serum homocysteine is associated with a $20 \%$ increased risk of circulatory health problems [13]. Elevated levels of homocysteine have been associated with endothelial dysfunction as a result of oxidative stress, inflammation, endoplasmic reticulum stress and increased levels of asymmetric dimethyl arginine, all of which can result in decreased concentration and bioavailability of endothelium-derived nitric oxide [14].

In the present study, a significant low mean level of serum vitamin B12 was present in CAD patients when compared with healthy controls $(242 \pm 98 \mathrm{pg} / \mathrm{ml}$ vs $333.7 \pm 197.1 \mathrm{pg} / \mathrm{ml})$. The mean serum folate level in CAD patient was lesser than in the healthy controls. (4.68 $\pm 3.6 \mathrm{vs}$ $5.03 \pm 3.37 \mathrm{ng} / \mathrm{ml}$ ) but the difference was not statistically significant. Similar findings were observed by Iqbal et al on analysis of role of B complex vitamins on hyperhomocysteinemia in patients with acute myocardial infarction [15]. A study by Dnyaneshwar Malharrao Ghuge et al, showed that statistical significance exists with increased serum homocysteine levels and decreased serum vitamin B12 levels among CHD groups when compared to healthy participants [16].Yan ma et al reported very low vitamin B12 levels among coronary artery disease patients who had high homocysteine levels compared to those with normal to low homocysteine concentration [1].

In our study the overall prevalence of vitamin B12 deficiency was found to be $33 \%$. Among the study participants, $50 \%$ were in the CAD group and $16 \%$ were in the control group. At the same time folic acid deficiency was not reported in any one of them. A case control study among patients with premature CAD in Tehran by Sadeghian et al showed that $26.6 \%$ and $10.7 \%$ of the study group had vitamin B12 and folic acid deficiencies respectively [7]. A study conducted in a Pakistani population of patients with acute coronary event showed vitamin B12 and folic acid deficiencies at $63.4 \%$ and $65.2 \%$ respectively [15]. Mahalle et al found vitamin B12 and folic acid deficiencies among CAD patients to be $86.7 \%$ and $2.7 \%$ respectively [17]. These variations may be attributed to regional differences, racial, ethnic, genetic factors and different lifestyles.

Several studies mainly the Stockholm Heart Epidemiology Program (SHEEP) [18] and The Kuopio Ischemic Heart Disease Risk Factor Study (KIHD) [19] have indicated the beneficial effects of B vitamins in cardiovascular protection. At the same time The Norwegian Vitamin Trial (NORVIT) [20], The Study of the Effectiveness of Additional Reductions in Cholesterol 
and Homocysteine trial (SEARCH) [19], The Western Norway B Vitamin Intervention Trial (WENBIT) [21] and Heart Outcomes Prevention Evaluation 2 Study (HOPE 2) [22] did not reveal lowered cardio vascular risk following vitamin B supplementation. The HOST study in which end stage renal disease patients received folic acid and vitamin B12 supplementation also showed no reduction in the incidence of CAD [23].

In our study we found the levels of vitamin B12 and folic acid to be lesser in males when compared to females but difference was found to be statistically significant only in relation to folic acid in the control group. Findings by Iqbal et al have shown decreased levels of both B12 and folate in males compared to females in both control and CAD group but this difference was statistically significant only with regards to vitamin B12 [15]. Naono et al in their analysis of vitamin B12 levels in patients who underwent percutaneous coronary intervention (PCI) from Korea also reported lowered levels of vitamin B12 in males as compared to females which is similar to the findings of our study [24]. On the contrary, Mahalle et al observed higher levels of vitamin B12 and folic acid among the male participants of their study [17].

All the participants in the control group were non-vegetarians and only one among the CAD group was a vegetarian. Development of Vitamin B12 deficiency is considered to be less prevalent on a non-vegetarian diet [25]. But Iqbal et al has reported high levels of vitamin B12 deficiency $(63.5 \%)$ in their study population which is mostly non vegetarian and has concluded that deficiency of vitamin B12 and folic acid are quite common in the population [15].

When we compared the mean values of various risk factors between the CAD patients and controls, we found that CAD patients had higher level of BMI, FPG, TC, TG, LDL-c and lower level of HDL-c than the controls. Our findings are similar to the study by Shabana et al,which analysed information regarding high BMI and dyslipidemic profile in the CHD group when compared with the healthy participants [26].

Vitamin B12 was found to be positively correlated with fasting plasma glucose, HDL-c, TC/HDL-c and LDL/HDL-c and folic acid and negatively correlated with TC, TGL, LDL-c, VLDL-c, RLP-c, Non-HDL-c, TG/HDL-c. Our results are in concordance with the findings observed by Mahalle et al [17] as well as Biswas et al [27] in their study involving the correlation of vitamin B12 deficiency with cardio vascular risk factors in CAD patients.

Logistic regression test showed vitamin B12 deficiency playing a vital role in development of risk factors in coronary artery disease $(\mathrm{OR}=5,95 \% \mathrm{CI}$ : $1.52-16.56 \mathrm{p}=0.0261)$ which is similar to the findings of Jacques et al [28].

Based on the angiographic findings of CAD patients, there was no association between the disease severity and vitamin B12 concentration. Brilakis et al during their evaluation of correlation between hyperhomocysteinemia and angiographic findings of CAD patients found no significant association [29].

The limitations of our study are its hospital-based nature, lesser number of female participants and the smaller sample size. Secondly estimation of homocysteine levels was not carried out. Low vitamin B12 and folic acid intake, as well as high homocysteine levels may be a contributing factor to the development of CAD. Therefore supplementation of vitamin B12 and folic acid may alleviate the risk for coronary artery disease. 


\section{Conclusion}

In our study the overall prevalence of vitamin B12 deficiency was found to be $33 \%$. Among this, $50 \%$ were in the CAD group and $16 \%$ were in the control group. Folic acid deficiency was not reported in any one of them. Among the participants $98 \%$ were on a non-vegetarian diet thus indicating that deficiency of vitamin B12 is commonly seen in the population. The levels of both vitamin B12 as well as folic acid were lesser in males as compared to females but this difference was statistically significant only in the control group with regards to folic acid. Vitamin B12 was also directly related to most of the traditional cardio vascular risk factors namely hyper-homocysteinemia and hyper-cholesterolemia. Therefore, supplementation of vitamin B12 and folic acid may help to reduce the incidence of coronary artery disease. Population based large scale studies can help to identify optimal cutoff values of vitamin B12 and folic acid as cardio protective factors.

\section{Acknowledgements}

We thank all the participants of the study. We gratefully acknowledge the support extended to us by Ortho Clinical Diagnostics for the analysis of vitamin B12 and folic acid.

\section{Conflict of interest}

The authors do not have any conflict of interest to declare.

\section{References}

1. Ma Y, Peng D, Liu C, Huang C, Luo J. "Serum high concentrations of homocysteine and low levels of folic acid and vitamin $B_{12}$ are significantly correlated with the categories of coronary artery diseases". BMC Cardiovasc Disord. Vol.17, no. 1, (2017), pp. 37.

2. Michael Lubran. "The Biochemistry of Folic Acid and Vitamin B12". Vol. 1. (1971).

3. Stanger O. "Physiology of Folic Acid in Health and Disease”. Curr Drug Metab. Vol.3, no. 2, (2005), pp. 211-23.

4. Djuric D, Jakovljevic V, Rasic-Markovic A, Djuric A, Stanojlovic O. "Homocysteine, folic acid and coronary artery disease: possible impact on prognosis and therapy”. Indian J Chest Dis Allied Sci. Vol.50, no. 1, (2008), pp. 39-48.

5. Doshi SN, McDowell IFW, Moat SJ, Lang D, Newcombe RG, Kredan MB, et al. "Folate improves endothelial function in coronary artery disease: An effect mediated by reduction of intracellular superoxide?". Arterioscler Thromb Vasc Biol. Vol.21, no. 7, (2001), pp. 1196202.

6. Brito A, Hertrampf E, Olivares M, Gaitán D, Sánchez H, Allen LH, et al. "Folate, vitamin B12 and human health”. Rev Med Chil. Vol.140, no. 11, (2012), pp. 1464-75.

7. Sadeghian S, Fallahi F, Salarifar M, Davoodi $G$, Mahmoodian M, Fallah N, et al. "Homocysteine, vitamin B12 and folate levels in premature coronary artery disease". BMC Cardiovasc Disord. Vol.6, (2006), pp. 1-7.

8. Gupta SK, Kotwal J, Kotwal A, Dhall A, Garg S. "Role of homocysteine \& MTHFR C677T gene polymorphism as risk factors for coronary artery disease in young Indians". Indian J Med Res. Vol.135, no. 4, (2012), pp. 506-12. 
9. Robinson K, Arheart K, Refsum H, Brattström L, Boers G, Ueland P, et al. "Low circulating folate and vitamin B6 concentrations risk factors for stroke, peripheral vascular disease, and coronary artery disease". Circulation. Vol.97, no. 5, (1998), pp. 437-43.

10. Tchantchou F. "Homocysteine metabolism and various consequences of folate deficiency". $J$ Alzheimers Dis. Vol.9, no. 4, (2006), pp. 421-7.

11. Clarke $R$, Armitage J. "Vitamin supplements and cardiovascular risk: Review of the randomized trials of homocysteine-lowering vitamin supplements". Seminars in Thrombosis and Hemostasis. Vol.26, (2000), pp. 341-8.

12. Humphrey LL, Fu R, Rogers K, Freeman M, Helfand M. "Homocysteine level and coronary heart disease incidence: A systematic review and meta-analysis". Mayo Clin Proc. Vol.83, no. 11, (2008), pp. 1203-12.

13. Roman Pawlak. "Is Vitamin B12 Deficiency a Risk Factor for Cardiovascular Disease in Vegetarians?". Am J Prev Med. Vol. 48, no. 6, (2015), pp. e11-e26.

14. Wang XC, Sun WT, Yu CM, Pun SH, Underwood MJ, He GW, et al. "ER stress mediates homocysteine-induced endothelial dysfunction: Modulation of IKCa and SKCa channels". Atherosclerosis. Vol.242, no. 1, (2015), pp.191-8.

15. Iqbal MP, Ishaq M, Kazmi KA, Yousuf FA, Mehboobali N, Ali SA, et al. "Role of vitamins B6, $B 12$ and folic acid on hyperhomocysteinemia in a Pakistani population of patients with acute myocardial infarction". Nutr Metab Cardiovasc Dis. Vol.15, no. 2, (2005), pp. 100-8.

16. Dnyaneshwar Malharrao Ghuge, Balasaheb Eknathrao Karad. "Study of correlation between serum vitamin B12 and homocysteine levels with ischemic heart disease". International Journal of Medicine. Vol. 16, no. 2, (2020), pp. 80-83.

17. Mahalle N, Kulkarni M V., Garg MK, Naik SS. "Vitamin B12 deficiency and hyperhomocysteinemia as correlates of cardiovascular risk factors in Indian subjects with coronary artery disease”. J Cardiol. Vol.61, no. 4, (2013), pp. 289-94.

18. Holmquist $C$, Larsson S, Wolk A, De Faire U. "Multivitamin supplements are inversely associated with risk of myocardial infarction in men and women - Stockholm Heart Epidemiology Program (SHEEP)'”. J Nutr. Vol. 133, no. 8, (2003), pp. 2650-4.

19. Voutilainen S, Rissanen TH, Virtanen J, Lakka TA, Salonen JT. "Low dietary folate intake is associated with an excess incidence of acute coronary events: The Kuopio Ischemic Heart Disease Risk Factor Study”. Circulation Vol.103, no. 22, (2001), pp. 2674-80.

20. Bonaa KH, Njølstad I, Ueland PM, Schirmer H, Tverdal A, Steigen T, et al. "Homocysteine Lowering and Cardiovascular Events after Acute Myocardial Infarction”. N Engl J Med. Vol.354, no. 15, (2006), pp. 1578-88.

21. Armitage JM, Bowman L, Clarke RJ, Wallendszus K, Bulbulia R, Rahimi K, et al. "Effects of homocysteine-lowering with folic acid plus vitamin B 12 vs placebo on mortality and major morbidity in myocardial infarction survivors: A randomized trial”. JAMA - J Am Med Assoc. Vol.303, no. 24, (2010), pp. 2486-94.

22. Ebbing M, Bleie O, Ueland PM, Nordrehaug JE, Nilsen DW, Vollset SE, et al. "Mortality and cardiovascular events in patients treated with homocysteine-lowering $B$ vitamins after coronary angiography: A randomized controlled trial". JAMA - J Am Med Assoc. Vol.300, no. 7, (2008), pp. 795-804.

23. Lonn E, Yusuf S, Arnold MJ, Sheridan P, Pogue J, Micks M, McQueen MJ, Probstfield J, Fodor $G$, Held C, Genest. "Heart Outcomes Prevention Evaluation (HOPE) 2 Investigators. 
Homocysteine lowering with folic acid and B vitamins in vascular disease". $N$ Engl J Med. Vol. 354, no. 15, (2006), pp. 1567-77.

24. Jamison RL, Hartigan P, Kaufman JS, Goldfarb DS, Warren SR, Guarino PD, et al. "Effect of homocysteine lowering on mortality and vascular disease in advanced chronic kidney disease and end-stage renal disease: A randomized controlled trial”. J Am Med Assoc. Vol.298, no. 10, (2007), pp. 1163-70.

25. Naono S, Tamura A, Kadota J. "Plasma homocysteine level is unrelated to long-term cardiovascular events in patients with previous percutaneous coronary intervention". $J$ Cardiol. Vol.54, no. 1, (2009), pp. 21-8.

26. Shabana, Saleem Ullah Shahid, and Sumbal Sarwar. "The abnormal lipid profile in obesity and coronary heart disease (CHD) in Pakistani subjects". Lipids Health Dis. Vol. 19, (2020), pp. 73.

27. Biswas S, Misra SK, Mukherjee P, Ghosh C. "Effect of dietary deficiency of vitamin B6, vitamin $B 12$ and folic acid on the development of coronary artery disease in adult Indian male patients". IOSR J Pharm Biol Sci. Vol.9, no. 6, (2014), pp. 30-5.

28. Jacques PF, Bostom AG, Wilson PWF, Rich S, Rosenberg IH, Selhub J. "Determinants of plasma total homocysteine concentration in the Framingham Offspring cohort". Am J Clin Nutr. Vol.73, no. 3, (2001), pp. 613-21.

29. Brilakis ES, McConnell JP, Ballman KV., Klee GG, Berger PB. "Lack of association between plasma homocysteine and angiographic coronary artery disease in the era of fortification of cereal grain flour with folic acid". Atherosclerosis. Vol.165, no. 2, (2002), pp. 375-81. 